\title{
BESCHREIBUNG EINES REGENERATIVEN ENERGIESYSTEMS MIT WASSERSTOFF- UND BRENNSTOFFZELLENTECHNIK FÜR LEHRE UND ANGEWANDTE FORSCHUNG
}

\author{
Denny Ragusch, Michael Jergović, Siegfried Rolle und Lutz B. Giese
}

\section{Zusammenfassung}

Angesichts der begrenzten Reichweiten fossiler Ressourcen und der durch ihren Verbrauch entstehenden Umweltprobleme, sind regenerative Energien notwendig für eine klimafreundliche Sicherstellung der Energieversorgung der Erde. Ein Hindernis für die breite Einführung regenerativer Energien ist, dass diese nicht grundlastfähig sind. Neue Speichertechnologien und komplexes Energiemanagement können hier Abhilfe schaffen. Die in dieser Veröffentlichung vorgestellte Anlage leistet hierzu einen Beitrag, insbesondere in der Ausbildung benötigter Ingenieure und Wirtschaftler. Kernkomponente dieser Anlage stellt die Wasserstoff- und Brennstoffzellentechnik zur klimafreundlichen Erzeugung, Speicherung und Rückverstromung von Wasserstoff dar. Mittels regenerativer Energien wird mit einem Elektrolyseur Wasserstoff erzeugt. Die Speicherung erfolgt in Metallhydridspeichern, welche sehr gut an das Druckniveau des Elektrolyseurs angepasst sind. Die Rückverstromung erfolgt mittels Brennstoffzellen. Es wird auf die praxisnahen Experimentiermöglichkeiten dieser Anlage, die ersten Betriebserfahrungen und auf zukünftige Forschungsthemen, eingegangen.

\section{Abstract}

Regarding the limited availability of fossil resources and the environmental impact by consuming them, thus Renewable Energy Sources (RES) are urgently needed to guarantee a climate-friendly global energy supply. However, while RES cannot supply the entire base-load, their introduction to the markets can be seen as handicapped. Smart energy storage and energy management systems may improve the situation. This publication is to demonstrate such a system which (i) is to be used to create scientific-technical progress and - as training equipment - (ii) may contribute in terms of educating engineers and scientists. Centerpiece of the appliance is the hydrogen and fuel-cell technology which is dedicated to generate and store hydrogen and furthermore to regain electricity in a climate-friendly way. Electrical energy from RES is transformed into hydrogen by a device called electrolyzer. The hydrogen is stored as hydrid in a metalhydrid-storage tank, which can be easily adapted to the gas-pressure level of the electrolyzer. In order to regain electricity, fuel-cells are to be used. This paper is to mention about (i) the experimental capabilities of the system in step with actual practice, (ii) first operational experience, and (iii) future fields of investigation and research.

\section{»I. EINFÜHRUNG UND MOTIVATION}

Fossile Energieträger wie Kohle, Erdöl und Erdgas weisen gravierende Nachteile auf. Ihr Verbrauch ist umweltschädlich und die Ressourcen sind begrenzt. Die Kernenergie mit Ihren nicht lösbaren Entsorgungs- und Gesundheitsproblemen stellt ebenso keine sinnvolle Alternative dar (Geitmann 2004). Die Sicherstellung der Energieversorgung der Menschen im 21. Jahrhundert kann auf umweltschonende Art nur mittels regenerativer Energiesysteme erfolgen. Problematisch ist, dass Wind- und Solarenergie nicht grundlastfähig sind (Wedding 2010). Aus diesem Grunde sind für die kurzund langfristige Speicherung überschüssiger Energiemengen aus den erneuerbaren Energien neue Speichertechnologien notwendig. Konventionelle Pumpspeicherwerke sind grundsätzlich geeignet, aber unter anderem durch die topografischen Gegebenheiten begrenzt. Eine Alternative bieten Speicherkonzepte basierend auf der Wasserstoff- und Brennstoffzellentechnik (Weltenergierat 2011). Weitere Vorteile von Wasserstoff als Energieträger sind unter anderem die Möglichkeit der dezentralen Herstellung, die $\mathrm{CO}_{2}$-Einsparung bei solarer Herstellung, Rückverstromung oder Verbrennung in modifizierten Verbrennungsmotoren, sowie die Kombination in Anlagen mit Kraft-WärmeKopplung. Die Wasserstoff- und Brennstoffzellentechnik wird unter anderem von der Bundesregierung über das Nationale Innovationsprogramm „Wasserstoff- und Brennstoffzellentechnologie“ gefördert, sodass hier von einem Wachstum der Branche auszugehen ist.

Aktuell und auch zukünftig werden sehr gut ausgebildete Ingenieure, Betriebswirtschaftler und angrenzende Berufe, die mit dieser Technik sowie den betriebswirtschaftlichen und rechtlichen Aspekten vertraut sind, benötigt. Daher wurde an der TH Wildau $[\mathrm{FH}]$ im Labor für regenerative Energietechnik ein regeneratives Energiesystem mit Wasserstoff- und Brennstoffzellentechnik für angewandte Forschung und Lehre aufgebaut, welches die breiten Anwendungsfelder Technik, Energiemanagement, Praxis, 
betriebswirtschaftliche und rechtliche Aspekte abdeckt. Hieraus folgen die Zielstellungen der Ausbildungsinhalte. Im Ingenieurwesen sind das beispielsweise die Anlagentechnik, das Systemdenken und die Sicherheitsaspekte im Umgang mit Wasserstoff. Als betriebswirtschaftliche Aspekte ergeben sich daraus z. B. Anlagenkosten, Service und Wartung bis hin zu den rechtlichen Aspekten. Diese Anlage ist geeignet für all diese Ausbildungsrichtungen- und inhalte, praktische Erfahrungen zu vermitteln.

\section{» II. SYSTEMBESCHREIBUNG}

Das regenerative Energiesystem mit Wasserstoff- Brennstoffzellentechnik wurde von der Firma Heliocentris errichtet und ist schematisch in der Abbildung 1 dargestellt. Der elektrische Teil ist als Inselsystem ausgelegt, bestehend aus Inselwechselrichter mit integriertem Batteriemanagement SMA Sunny Island SI5048, mit einem Batteriespeicher $4 \times$ Hoppecke SB120 mit einer Akkusystemspannung $V_{D C}=48 \mathrm{~V}$ und einer Speicherkapazität von $\mathrm{Q}_{\text {Akku}}=120$ Ah zur Generierung einer einphasigen $230 \mathrm{~V}$ Wechselspannung, an welcher wechselspannungsseitig regenerative Energiequellen (Fotovoltaik Solarwatt $P_{\text {Sonne }}=1100 \mathrm{Wp}$, Windkraftanlage Venco Twister 300T mit $P_{\text {Wind }}=300 \mathrm{Wp}$ und Brennstoffzellen $4 \times$ Heliocentris Nexa 1200 mit insgesamt $P_{B Z}=4,8 \mathrm{~kW}$, welche einzeln zugeschaltet werden können) über entsprechende Wechselrichter SMA SunnyBoy, SMA WindyBoy und SMA
Hydroboy angeschlossen sind. Neben dem Akkumulator als Energiespeicher ist ein zukunftsweisendes Energiespeichersystem, basierend auf Wasserstoff als Energieträger, integriert. Dieses besteht aus einer Wasseraufbereitungsanlage, einem Elektrolyseur für die Wasserstofferzeugung, einem Metallhydridspeichersystem für die Wasserstoffspeicherung und den Brennstoffzellen für die Rückverstromung von Wasserstoff. Der PEM-Elektrolyseur Hogen S40 der Firma Proton Energy Systems kann maximal $1 \mathrm{Nm}^{3}$ Wasserstoff pro Stunde produzieren bei 7,36 kW elektrischer Leistung. Die Wasserstoffreinheit beträgt 99,9995\%. Der Wasserstoffspeicher besteht aus zwei Wasserstoffspeicherbänken mit jeweils 3 Metallhydridspeichern MHS1000IHE der Firma Treibacher Industrie AG. Das gesamte Speichervolumen beträgt ca. 23.400 Normliter Wasserstoff. Der maximale Beladedruck der verwendeten Metallhydridspeicher beträgt 30 bar. Prinzip bedingt - und für Forschungszwecke in einem erweiterten Bereich - können die Wasserstoffspeicher gekühlt (bei der Wasserstoffspeicherung) oder beheizt (bei der Wasserstoffentnahme) werden. Alternativ und für verschiedenste Fragestellungen können an der Anlage 200 bar Wasserstoffdruckgasflaschen verwendet werden. Weitere Bestandteile der Gesamtanlage sind Umweltund Laborsensorik zur Bestimmung der Windgeschwindigkeit, der Intensität der Sonneneinstrahlung, der Temperaturen von ausgewählten Solarmodulen, Lufttemperatur und Luftfeuchte in der Umwelt und im Labor,

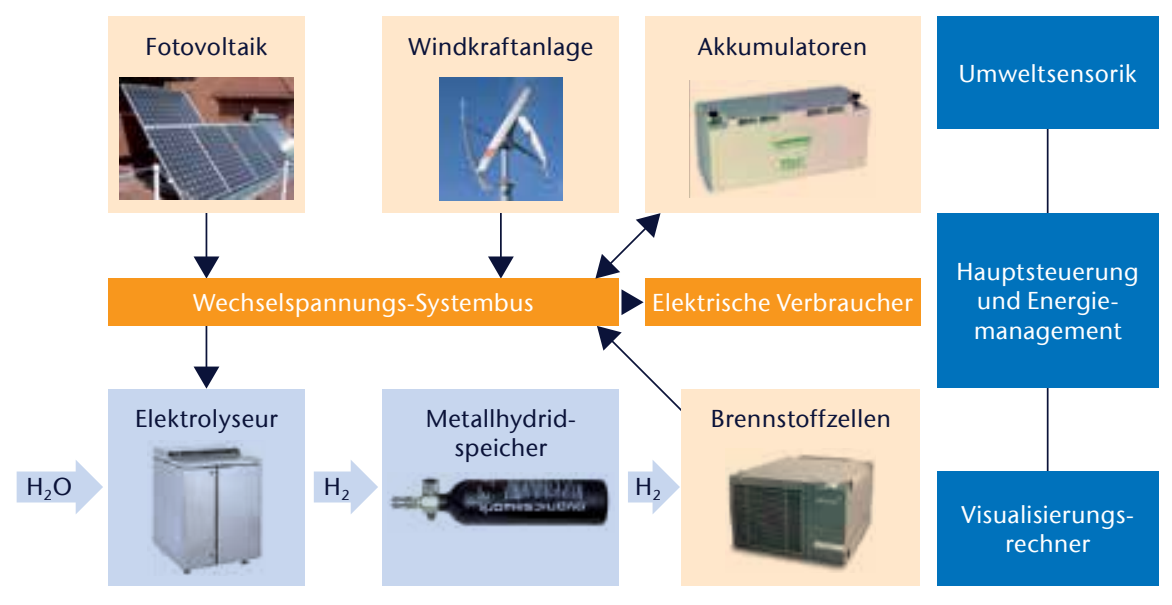

Abb. 1) Schematische Darstellung des regenerativen Energiesystems mit Wasserstoff-Brennstoffzellentechnik

Wasserstoffsensoren für die Sicherheit, mehrere Druck- und Durchflusssensoren in der Anlage zur Bestimmung des Anlagenzustandes im zeitlichen Verlauf. Das System ist modular aufgebaut und kann um verschiedene Komponenten erweitert werden wie beispielsweise zusätzlicher Fotovoltaik- oder Windkraftanlagen, Brennstoffzellen, aber auch BHKWs oder Wärmepumpen für die Nutzung thermischer Energie.

Alle Systeme sind über eine Industriesteuerung (Siemens Simatic S7-300) kommunikativ verbunden, die gesamten Anlagenparameter und Messwerte werden in einer MySQL-Datenbank abgelegt und werden über einen OPC-Server bereitgestellt. Die Vernetzung ermöglicht eine intelligente Regelung und Steuerung der einzelnen Komponenten bis hin zum Abschalten einzelner Verbraucher. Verschiedene Regelungsstrategien für das Energiemanagement können direkt über die Betriebsführungssoftware an der Anlage getestet und visualisiert werden. Über die Visualisierungssoftware werden die einzelnen Experimente gestartet und die Anlage sicher gesteuert. Eine Fehlbedienung der Anlage ist somit ausgeschlossen.

\section{» III. EXPERIMENTIERMÖGLICHKEITEN}

Die Betriebsführungssoftware der Anlage ermöglicht die Untersuchung der einzelnen Subsysteme und des Gesamtsystems, insbesondere der Wasserstofferzeugung inklusive Wasseraufbereitung mittels Elektrolyseur, der Wasserstoffspeicherung, der Rückverstromung von Wasserstoff mittels Brennstoffzellen, dem Betrieb als Inselstromnetz und des automatischen Energiemanagementsystems (EMS). Für die eben genannten Bereiche gibt es in der Betriebsführungssoftware vorbereitete Experimente, mit denen die Anlage gesteuert und wichtige Anlagenparameter visualisiert werden. Die Abbildung 2 zeigt ein Bildschirmfoto der Softwareoberfläche mit dem aktivierten Reiter „Übersicht“, auf dem die gesamte Anlage mit all Ihren Systemkomponenten dargestellt ist. Bei Anwahl eines Experimentes 
werden nicht benötigte Anlagenkomponenten ausgeblendet und nur die jeweils für das Experiment benötigten Anlagenparameter sind verfügbar. Detailinformationen zu einzelnen Komponenten können zusätzlich abgerufen werden.

Für weitergehende komplexere Untersuchungen, die mit den vorbereiteten Experimenten nicht durchgeführt werden können, besteht die Möglichkeit, über den Zugriff auf OPC-Server und MySQL-Datenbank die Anlage innerhalb der Systemgrenzen zu steuern, und auf die aufgezeichneten Messdaten zuzugreifen.

\section{»IV. ERSTE ERGEBNISSE}

Die Inbetriebnahme wurde nach baubedingter Unterbrechung (Gebäudebrandschutz) und daraus folgenden Anlaufschwierigkeiten, wegen Stillstand von Komponenten, verzögert. Sowohl die einzelnen Systemkomponenten als auch die Subsysteme wurden erfolgreich auf ihre Funktionstüchtigkeit und ihr komplexes Zusammenspiel untersucht. Die Wasserstoffspeicher wurden mit dem vom Elektrolyseur erzeugten Wasserstoff beladen. Der gespeicherte Wasserstoff wurde mittels Brennstoffzellen in elektrische Energie und Wärme umgewandelt. Verschiedene Verbraucher wurden mit elektrischer Energie aus dem Inselnetz versorgt und grundlegende Energiemanagement-Funktionen getestet. Das vorhandene Sicherheitssystem wurde umfassend geprüft und arbeitet innerhalb der Spezifikationen.

\section{» V. ZUSAMMENFASSUNG UND AUSBLICK}

Zur Lösung der Energieproblematik des 21. Jahrhunderts sind sehr gut ausgebildete Ingenieure, Betriebswirtschaftler und angrenzende Berufe nötig. Für einen Teil dieser Ausbildung und zu Forschungszwecken wurde ein bei Bedarf leicht erweiterbares regeneratives Energiesystem mit Wasserstoff- und Brennstoffzellentechnik im Labor für regenerative Energietechnik errichtet. Dieses System und die Experimentiermöglichkeiten wurden im Detail vorgestellt, Hauptmerkmal ist die klimafreundliche und $\mathrm{CO}_{2}$-neutrale Erzeugung von Wasserstoff mittels Elektrolyseurs über regenerative Energien sowie der Speicherung und Rückverstromung des Wasserstoffes. Die Anlage wurde erfolgreich in Betrieb genommen.
Forschungsgegenstände werden unter anderem die Praxistauglichkeit der einzelnen Systemkomponenten als auch das Zusammenspiel des komplexen Gesamtsystems sein. Hierzu gehören sowohl technische als auch wirtschaftliche und rechtliche Aspekte. Für den Bereich Energiemanagement und Energieeffizienz ist geplant, über Bachelor- und Masterarbeiten Modelle der einzelnen Systemkomponenten und komplexe Regelungsstrategien zu entwickeln und diese auf Wirtschaftlichkeit zu untersuchen. Mit der vorgestellten Anlage und der im Labor vorhandenen Solarthermieanlage kann insbesondere das zukunftsweisende Beispiel Energieautarkes-Einfamilienhaus abgebildet und experimentell untersucht werden.

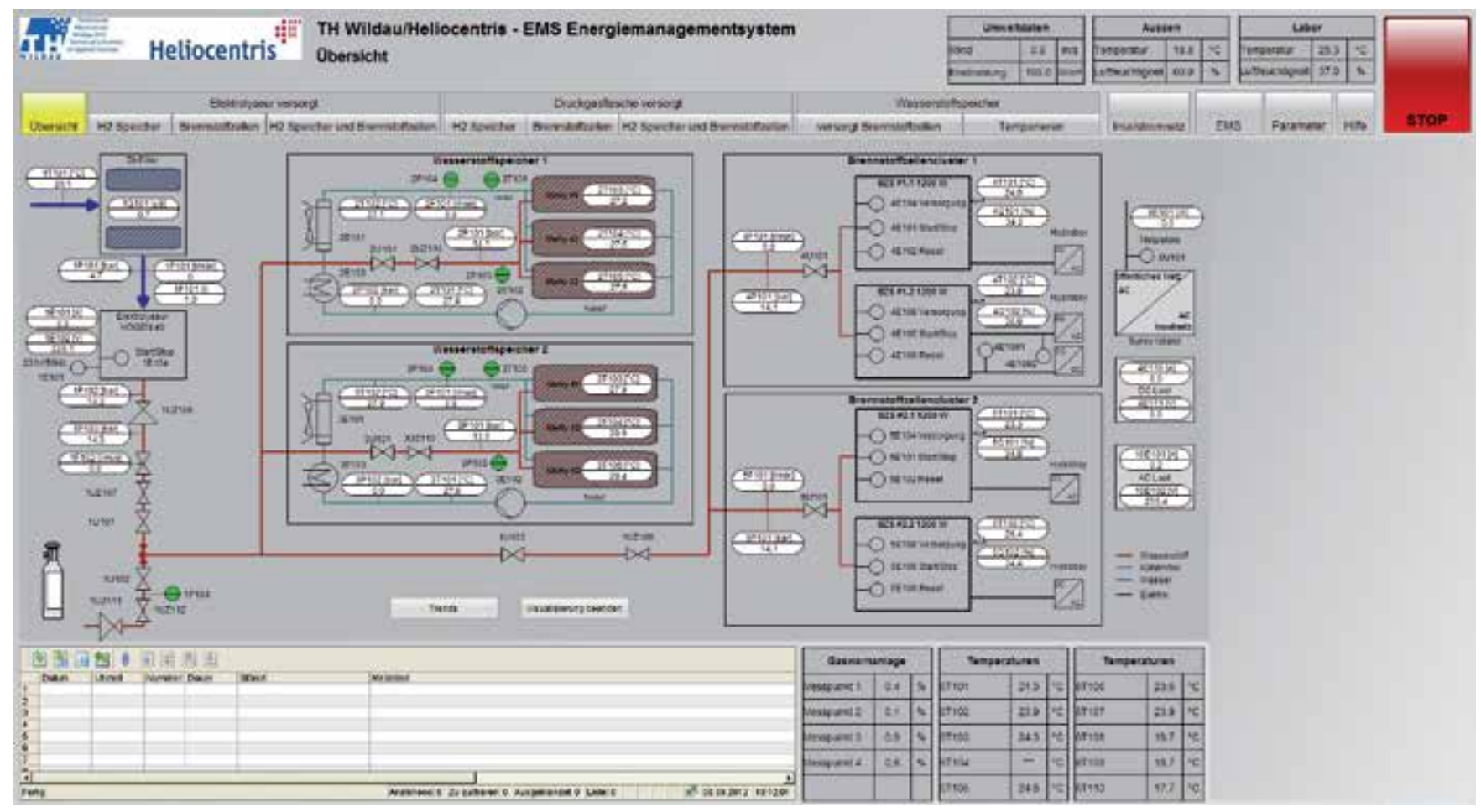

Abb. 2) Bildschirmfoto der Betriebsführungssoftware mit aktiviertem Reiter „Übersicht“ 


\section{LITERATUR}

Geitmann, Sven (2004): Wasserstoff \& Brennstoffzellen Die Technik von Morgen.

Wedding, Steven Jörg (2010): Inbetriebnahme eines regenerativen Energiesystems mit Wasserstoff und

Brennstoffzellentechnik.

Weltenergierat - Deutschland e.V. (2011): Energie für

Deutschland 2011 - Fakten, Perspektiven und Positionen

im globalen Kontext, Schwerpunktthema: Integration

erneuerbarer Energien in das Stromversorgungssystem.

\section{AUTOREN}

M. Eng. Dipl.-Ing. [FH] Denny Ragusch

Labor für Regenerative Energietechnik

TH Wildau [FH]

denny.ragusch@th-wildau.de

Dipl.-Ing. Michael Jergović

Labor für Regenerative Energietechnik

TH Wildau [FH]

michael.jergovic@th-wildau.de

Prof. Dr. rer. nat. Siegfried Rolle

Labor für Regenerative Energietechnik

TH Wildau [FH]

siegfried.rolle@th-wildau.de

Dr. rer. nat. Lutz B. Giese

Labor für Regenerative Energietechnik

TH Wildau [FH]

lutz.giese@th-wildau.de 